\title{
BMJ Open Minimally invasive versus conventional sternotomy for Mitral valve repair: protocol for a multicentre randomised controlled trial (UK Mini Mitral)
}

\author{
Rebecca H Maier (D) , ${ }^{1}$ Adetayo S Kasim (D) , ${ }^{2}$ Joseph Zacharias, ${ }^{3}$ Luke Vale (D) , ${ }^{4}$ \\ Richard Graham, ${ }^{5}$ Antony Walker, ${ }^{3}$ Grzegorz Laskawski, ${ }^{3}$ Ranjit Deshpande, ${ }^{6}$ \\ Andrew Goodwin, ${ }^{5}$ Simon Kendall, ${ }^{5}$ Gavin J Murphy (1) , ${ }^{7}$ Vipin Zamvar (D) , \\ Renzo Pessotto, ${ }^{8}$ Clinton Lloyd, ${ }^{9}$ Malcolm Dalrymple-Hay, ${ }^{9}$ Roberto Casula, ${ }^{10}$ \\ Hunaid A Vohra, ${ }^{11}$ Franco Ciulli, ${ }^{11}$ Massimo Caputo (D) , ${ }^{12}$ Serban Stoica, ${ }^{11}$ \\ Max Baghai, ${ }^{6}$ Gunaratnam Niranjan, ${ }^{13}$ Prakash P Punjabi, ${ }^{10}$ Olaf Wendler, ${ }^{6}$ \\ Leanne Marsay, ${ }^{1}$ Cristina Fernandez-Garcia (D) , ${ }^{4}$ Paul Modi, ${ }^{14}$ Bilal H Kirmani, ${ }^{14}$ \\ Mark D Pullan, ${ }^{14}$ Andrew D Muir, ${ }^{14}$ Dimitrios Pousios, ${ }^{14}$ Helen C Hancock (1) , ${ }^{1}$ \\ Enoch Akowuah (D) ${ }^{5}$
}

To cite: Maier RH, Kasim AS, Zacharias J, et al. Minimally invasive versus conventional sternotomy for Mitral valve repair: protocol for a multicentre randomised controlled trial (UK Mini Mitral). BMJ Open 2021;11:e047676. doi:10.1136/ bmjopen-2020-047676

- Prepublication history for this paper is available online. To view these files, please visit the journal online (http://dx.doi. org/10.1136/bmjopen-2020047676).

$\mathrm{HCH}$ and EA are joint senior authors.

Received 09 December 2020 Revised 10 February 2021 Accepted 12 March 2021

\section{Check for updates}

(C) Author(s) (or their employer(s)) 2021. Re-use permitted under CC BY. Published by BMJ.

For numbered affiliations see end of article.

Correspondence to Rebecca H Maier; Rebecca.Maier@newcastle. ac.uk

\section{ABSTRACT}

Introduction Numbers of patients undergoing mitral valve repair (MVr) surgery for severe mitral regurgitation have grown and will continue to rise. MVr is routinely performed via median sternotomy; however, there is a move towards less invasive surgical approaches.

There is debate within the clinical and National Health Service (NHS) commissioning community about widespread adoption of minimally invasive MVr surgery in the absence of robust research evidence; implementation requires investment in staff and infrastructure.

The UK Mini Mitral trial will provide definitive evidence comparing patient, NHS and clinical outcomes in adult patients undergoing MVr surgery. It will establish the best surgical approach for MVr, setting a standard against which emerging percutaneous techniques can be measured. Findings will inform optimisation of cost-effective practice.

Methods and analysis UK Mini Mitral is a multicentre, expertise based randomised controlled trial of minimally invasive thoracoscopically guided right minithoracotomy versus conventional sternotomy for MVr. The trial is taking place in NHS cardiothoracic centres in the UK with established minimally invasive mitral valve surgery programmes. In each centre, consenting and eligible patients are randomised to receive surgery performed by consultant surgeons who meet protocol-defined surgical expertise criteria. Patients are followed for 1 year, and consent to longer term follow-up.

Primary outcome is physical functioning 12 weeks following surgery, measured by change in Short Form Health Survey (SF-36v2) physical functioning scale. Early and 1 year echo data will be reported by a core laboratory. Estimates of key clinical and health economic outcomes will be reported up to 5 years.

The primary economic outcome is cost effectiveness, measured as incremental cost per quality-adjusted life year gained over 52 weeks following index surgery.

\section{Strengths and limitations of this study}

Largest randomised controlled trial of minimally invasive cardiac surgery globally.

- Strong and ongoing patient and public involvement and international clinical consensus informed trial design.

Robust design, with 90\% power, includes randomisation to surgery performed by consultant surgeons with defined expertise beyond the learning curve. Design requires some patients to move between surgeons, which may impact recruitment but assures quality of outcomes and reduces bias.

- Multicentre UK trial with blinding of central primary outcome assessor and echocardiogram core laboratory.

Up to 5 year estimates of key clinical and survival outcomes.

Ethics and dissemination A favourable opinion was given by Wales REC 6 (16/WA/0156). Trial findings will be disseminated to patients, clinicians, commissioning groups and through peer reviewed publication.

Trial registration number ISRCTN13930454.

\section{INTRODUCTION}

Mitral regurgitation is most commonly caused by degenerative mitral valve disease, which leads to dilatation of the mitral valve annulus, leaflet prolapse or leaflet restriction. It can also be caused by rheumatic valve disease, or infective endocarditis.

Mitral valve repair (MVr) surgery is the optimal treatment for patients with severe mitral regurgitation caused by degenerative 
disease; when compared with mitral valve replacement, it carries a lower perioperative mortality, improved survival, better preservation of left ventricular function and lower long-term morbidity and mortality. ${ }^{1-6}$

MVr surgery for mitral valve regurgitation is frequently performed ${ }^{7}$ and patient numbers in the UK increased by a third between 2003 and 2012 (from 1549 to $2118^{89}$; the number of patients undergoing isolated MVr surgery more than doubled over the same time (681 in 2003 to 1456 in 2012)). ${ }^{89}$ The rise is expected to continue. Furthermore, international guidance recommends that asymptomatic patients may benefit from early surgery. ${ }^{10}$

\section{Choice of comparators}

Mitral valve surgery is routinely performed via sternotomy which involves dividing the sternum completely, enabling easy access to the heart and cannulation of the great vessels centrally to establish cardiopulmonary bypass. It allows flexibility in myocardial protection strategies and potentially simplifies deairing and haemostasis at the end of the procedure.

Disadvantages of a sternotomy incision include an increase in bleeding because of the size of the incision. ${ }^{11}$ The mitral valve is posterior to the incision and valve access can be difficult. Wound infections occur in 2\%-3\% of patients, and significant morbidity, and mortality, can result from this complication. ${ }^{12}{ }^{13}$ The sternotomy incision is usually closed using multiple stainless steel wires facilitating immobilisation of the sternum while sternal union occurs. It can take up to 3 months for the sternum to heal completely. ${ }^{12}$ During this period, the activity of patients is significantly limited to reduce the risk of sternal dehiscence. These limitations can reduce the speed of recovery and limit patients' ability to return to usual activities. Moreover, limited activity during this period can increase the risk of complications in the recovery period.

Minimally invasive approaches are increasingly used in all surgical specialties. In cardiac surgery, they are routinely used for coronary revascularisation ${ }^{14}$ aortic valve surgery, aortic root surgery ${ }^{15}$ and surgery for assist devices. ${ }^{16}$

Several publications have established the safety of minimally invasive mitral valve surgery. ${ }^{17-22}$ Outcomes in a cohort of 1000 patients undergoing minimally invasive mitral valve surgery between 2003 and 2011 demonstrated a mortality of $0.7 \%$ at 1 year, and 8 year survival of $90 \% .^{18}$ Of those surviving, freedom from reoperation at 8 years was $93 \% .^{18}$ The approach may also significantly reduce morbidity and mortality in high-risk patients including in the elderly. ${ }^{19-21}$ A propensity matched analysis of 2400 cases from 3 UK centres suggested a reduction of blood transfusion $(20.5 \%$ vs $14.4 \%, \mathrm{p}=0.005)$ and median length of hospital stay ( 7 vs 6 days, $\mathrm{p}<0.001$ ) when minimally invasive surgery was compared with conventional surgery despite longer procedural times. ${ }^{22}$

There is emerging evidence from non-NHS healthcare settings that the minimally invasive approach is less costly than conventional sternotomy; with cost savings driven by reduced intensive care and hospital stay as well as reduced need for blood transfusion. ${ }^{23}$

\section{Current evidence supporting the rationale for the trial}

Five published meta-analyses compare minimally invasive with conventional MVr. ${ }^{24-28}$ These identify only two small randomised controlled trials (RCTs) reporting short-term ${ }^{29}$ and long-term ${ }^{30}$ outcomes and base their main conclusions on evidence from observational studies; ${ }^{18} 1923$ 31-38 these provide no evidence of differences in clinical outcomes. The literature is severely limited by the absence of robust data on comparative outcomes between the two techniques. ${ }^{24-28}$

The International Society of Minimally Invasive Cardiac surgery published a consensus document on the role of minimally invasive mitral valve surgery in contemporary cardiac surgery practice in $2010 .^{39}$ This highlights the limitations of available evidence in reaching a consensus, particularly the lack of adequately powered prospective RCTs to establish the comparative effectiveness of the two approaches. Its recommendation was for further prospective RCTs, adequately powered to assess quality of life, complications, efficacy (repair rates) and clinically relevant outcomes, particularly long term survival, patient functionality and freedom from re-operation. ${ }^{39}$

Less than $5 \%$ of patients having mitral valve surgery in the UK currently have a minimally invasive MVr, largely as a result of the lack of clear and definitive evidence. There is consensus in the cardiac surgery community that the optimum surgical approach to treat these patients urgently needs defining. This is particularly pressing as there is emerging evidence relating to the effectiveness of percutaneous MVr with the mitraclip and other devices in degenerative ${ }^{40}$ and functional mitral regurgitation. ${ }^{41}$ It is now clear that trials of percutaneous approaches and surgery are needed, but cannot be undertaken until the optimal surgical approach is clearly defined.

\section{METHODS AND ANALYSIS \\ Study design}

UK Mini Mitral is a multicentre, RCT of minimally invasive thoracoscopically-guided right minithoracotomy (intervention) versus conventional sternotomy (control) in patients undergoing MVr. The trial includes an internal pilot to assess the likelihood of meeting recruitment targets.

The trial will answer the questions 'Are improvements in physical functioning and associated return to usual activities seen in patients who undergo minimally invasive mitral valve surgery compared with conventional surgery?' and 'Is minimally invasive mitral valve surgery compared with conventional surgery cost-effective?'

\section{Setting}

Three hundred and sixteen patients due to receive MVr surgery will be recruited from UK NHS cardiothoracic surgery units with established minimally invasive and 
conventional mitral valve services. All centres will be able to accommodate the needs of this trial including established minimally invasive MVr services, research nurse and echocardiographer support, and facilities to carry out trial assessments. Only units in equipoise in the way they manage their patients requiring MVr will participate.

At each centre, individual surgeons perform one type of operation; $\mathrm{MVr}$ via thoracoscopically guided right minithoracotomy or MVr via median sternotomy. Before performing surgery within the trial, each surgeon has completed 50 of these; the Trial Steering Committee (TSC) reviews a record of the number of operations for each surgeon, and agrees to their participation, in advance of them doing so. Depending on their allocation, patients can be required to move to another surgeon following randomisation.

\section{Eligibility criteria}

Patients are eligible to take part if they are scheduled to have surgery for MVr and fulfil all eligibility criteria:

\section{Inclusion criteria}

- Adult ( $\geq 18$ years old at consent) with degenerative mitral valve disease, requiring $\mathrm{MVr}$ *

- Written informed consent.

- Fit for cardiac surgery and cardiopulmonary bypass.

*Patients requiring concomitant surgery for atrial fibrillation (AF), tricuspid valve repair, and/or patent foramen ovale (PFO) closure are included

\section{Exclusion criteria}

- Concomitant cardiac surgery other than for AF, tricuspid valve repair and PFO closure.

- Requiring mitral valve replacement.

- Acute (current) infective endocarditis.

- Emergency or salvage surgery.

- Only one surgical technique indicated.

- Pregnant.**

- Currently participating in another interventional clinical trial.

- $\geq 4$ weeks as an inpatient prior to randomisation.

- Previous cardiac surgery.

- Mobility impairments that would preclude Short Form Health Survey (SF36-v2) completion.

**Female patients between the ages of 18 and 50 will receive a pregnancy test at baseline.

\section{Randomisation}

Eligible patients will be randomised in a 1:1 ratio to undergo $\mathrm{MVr}$ using minimally invasive thoracoscopically guided right minithoracotomy (intervention under study) or conventional median sternotomy (control arm/ usual care) by members of the research team at each centre using a 24-hour, central, secure, web-based randomisation system with concealed allocation (procured from Sealed Envelope).

Randomisation is performed using a minimisation scheme, which adjusts for baseline SF-36v2 physical functioning score, presence or absence of AF and/or $\mathrm{PFO}$, and presence and severity of tricuspid regurgitation.

\section{Trial surgical interventions}

The intervention arm will receive MVr via minimally invasive thoracoscopically guided right minithoracotomy. The control group receive $\mathrm{MVr}$ via conventional sternotomy.

\section{Intervention arm}

The patient is intubated with a single or double lumen endotracheal tube. Cardiopulmonary bypass is established by femoral artery cannulation and venous return achieved from the venae cavae using a single bicaval cannula placed from the femoral vein, or with an additional cannula in the superior venae cava. Transoesophageal echocardiography confirms the optimum location of the venous and arterial cannulas.

A $4-7 \mathrm{~cm}$ right lateral minithoracotomy is then used to enter the thorax through the third or fourth intercostal space. A soft-tissue retractor, with or without a small thoracic retractor, is used to spread the ribs. The pericardium is opened $3-4 \mathrm{~cm}$ anterior and parallel to the phrenic nerve from the distal ascending aorta to the diaphragm. A video camera is inserted through a 5-10 mm port.

Endoballoon occlusion, or a transthoracic clamp, achieves aortic occlusion. Cardiac arrest is achieved with repeated doses of cardioplegia. The mitral valve is approached through a paraseptal incision and a left atrial retractor is used to expose the mitral valve.

Following the mitral valve procedure, the left atrium is closed, the heart deaired and aortic occlusion removed. Cardiopulmonary bypass is then discontinued and the thoracotomy incision closed once haemostasis has been achieved.

\section{Control arm}

The sternum is divided completely (from the collarbone to the bottom of the breastbone) and cardiopulmonary bypass established by siting cannulas in the right atrium and inferior venae cava and ascending aorta.

Cardiac arrest is achieved with cardioplegia and the mitral valve approached via the left atrium. The valve is repaired and assessed intraoperatively by water testing. Once the repair is deemed satisfactory, the atrium is closed, deairing manoeuvres performed, and the aortic cross clamp removed to allow reperfusion of the heart. Cardiopulmonary bypass is discontinued once haemostasis is achieved and the sternum is closed.

\section{Outcomes}

The primary outcome is physical functioning and associated return to usual activities measured by change in SF-36v2 physical functioning scale ${ }^{42} 12$ weeks following index surgery using a 4-week recall period.

The primary economic outcome is cost effectiveness measured in terms of incremental cost per qualityadjusted life year (QALY) gained over 52 weeks following index surgery. 
Secondary outcomes post index MVr:

- Feasibility and study recruitment, explored by means of a 6-month internal pilot.

- Cardiac function; assessed using transthoracic echocardiography (TTE), early (1 day to 12 weeks) and late (52 weeks) by an independent core laboratory.

- Mitral valve and mitral valve surgery related events and survival (morbidity and mortality); compared at 52 weeks and up to 5 years using adverse event data and Hospital Episode Statistics and National Institute for Cardiac Outcomes Research data.

- Physical functioning and quality of life over 52 weeks; compared using SF-36v2 and EuroQol 5 Dimension 5 Level (EQ-5D-5L). ${ }^{42-45}$

- Levels of physical activity and quality of sleep; quantified using accelerometer measures (GENEActiv ${ }^{46-50}$ ) over 52 weeks.

- Surgical outcomes quantified over 52 weeks.

- Costs, including intervention-specific estimates, of the two operations and their sequalae over 52 weeks.

- QALYs, estimated from EQ-5D-5L $\mathrm{L}^{43-45}$ and SF-6D (derived from the SF-36v2) over 52 weeks.

- Modelled costs and QALYs over the patient lifetime.

- Modelled incremental cost per QALY gained over the patient lifetime

- Hospital Episode Statistics (HES) data will be explored to understand if it adequately captures mitral valve events.

\section{Sample size}

The primary outcome measure is change in physical functioning scale within SF-36v2 ${ }^{42}$ from baseline to 12 weeks following index surgery. On the basis of the literature and consulting with cardiac and patient communities, a minimally clinically important difference is 10 points on the scale. UK Mini Mitral is powered to investigate the superiority of thoracoscopically guided right minithoracotomy over conventional sternotomy.

One study $^{31}$ reports a small variation (SD of 8 ) in SF-36 physical function scores; this is not seen in other studies ${ }^{3233}$ which report a SD of 30 . We used the conservative estimate to inform the original sample size calculation:

Assuming alpha of $5 \%$ and $90 \%$ power, 382 patients (191 in each arm) would be required to detect a minimally clinically important difference of 10 points in the SF-36v2 ${ }^{42}$ physical functioning scale at 12 weeks (assuming SD of 30); allowing for attrition, we planned to randomise 400 patients. To assess our assumptions, we performed a blinded sample size re-estimation using baseline SF-36v2 physical functioning scale data from 177 trial patients. Using the re-estimated SD of 26.3 with $90 \%$ power, 288 patients are required to detect a 10 point difference in SF-36v2 physical functioning at 12 weeks. Accounting for $10 \%$ attrition, 316 patients will be recruited.

\section{Trial procedures}

Patients due to undergo isolated MVr surgery at participating centres are identified at the point of referral (elective patients) and from the inpatient waiting list (urgent but non-emergency patients) by the clinical research team and approached about the trial, including given an information sheet and consent form. After discussion, consent is sought, baseline assessments performed and eligibility checked and confirmed by one of the consultant surgeons participating in the trial. Eligible patients are randomised, and their general practitioners informed. A full schedule of events is detailed in table 1 , and a participant flow chart provided in figure 1 . Patients have assessments at 6,12, 18, 24, 38 and 52 weeks following index surgery. Longer term follow-up will involve routinely collected data.

\section{SF-36v2 and EQ-5D-5L}

Physical functioning and overall quality of life are assessed using SF-36v2 and EQ-5D-5L ${ }^{42-4551}$ questionnaires administered prior to randomisation. Questionnaire completion is repeated over the telephone at $6,12,18,24,38$ and 52 weeks following index surgery.

\section{Echocardiogram}

Cardiac function is assessed via TTE at baseline, post operatively ( 1 day to 12 weeks) and at 52 weeks. Measurements of left ventricular volumes, dimensions and function, mitral regurgitation severity and estimates of right heart function and pulmonary artery pressure are reported.

\section{Accelerometer}

Levels of physical activity and quality of sleep are quantified using accelerometer measures. Accelerometers are worn on patients' non-dominant wrist nonstop for 7 consecutive days at seven timepoints (baseline, 6, 12, 18, 24, 38 and 52 weeks following index surgery). The restrictions of movement by being an inpatient means accelerometer measures are not possible for inhouse urgent patients and are not to be undertaken.

\section{Statistical analysis}

Primary analysis will follow intention to treat principles, with patients analysed according to randomisation, irrespective of surgical intervention received. A full statistical analysis plan will be developed and agreed with the Independent Data Monitoring and Ethics Committee (IDMEC) and TSC prior to the end of data collection.

Outcome data will be analysed at the end of the study; no interim outcome analyses are planned.

Primary analysis of change in physical functioning, measured using SF-36v2 at 12 weeks, will use a general linear model accounting for surgical intervention, baseline scores, valve pathology and concomitant surgery. Robust SE will account for intrapatient correlation due to repeated measures. A similar modelling approach will be used for the final analysis of change in physical functioning at 52 weeks.

Secondary outcomes: continuous outcomes will be analysed using a general linear model, binary outcomes will be analysed using a generalised estimating equation to account for repeated binary data over time, categorical 
Table 1 UK Mini Mitral Schedule of Events

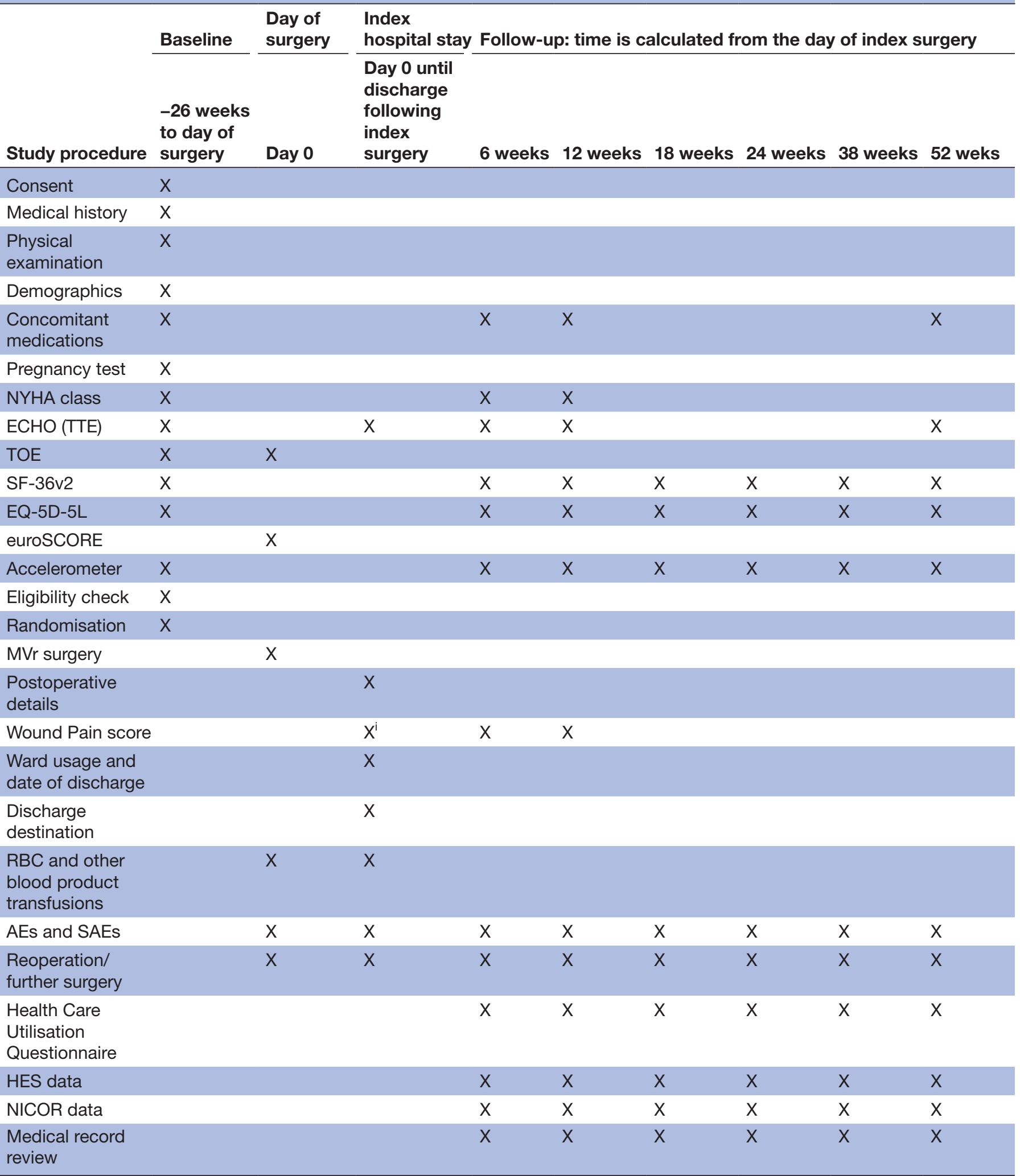

AE, adverse event; ECHO, Echocardiogram; EQ-5D-5L, EuroQol 5 Dimension 5 Level; HES, Hospital Episode Statistics; MVr, mitral valve repair; NICOR, National Institute for Cardiac Outcomes Research; NYHA, New York Heart Association; RBC, Red Blood Celleuor; SAE, serious adverse event; SCORE, European System for Cardiac Operative Risk Evaluation; SF36-v2, Short Form Health Survey; TOE, transoesophageal echocardiography; TTE, transthoracic echocardiography. 


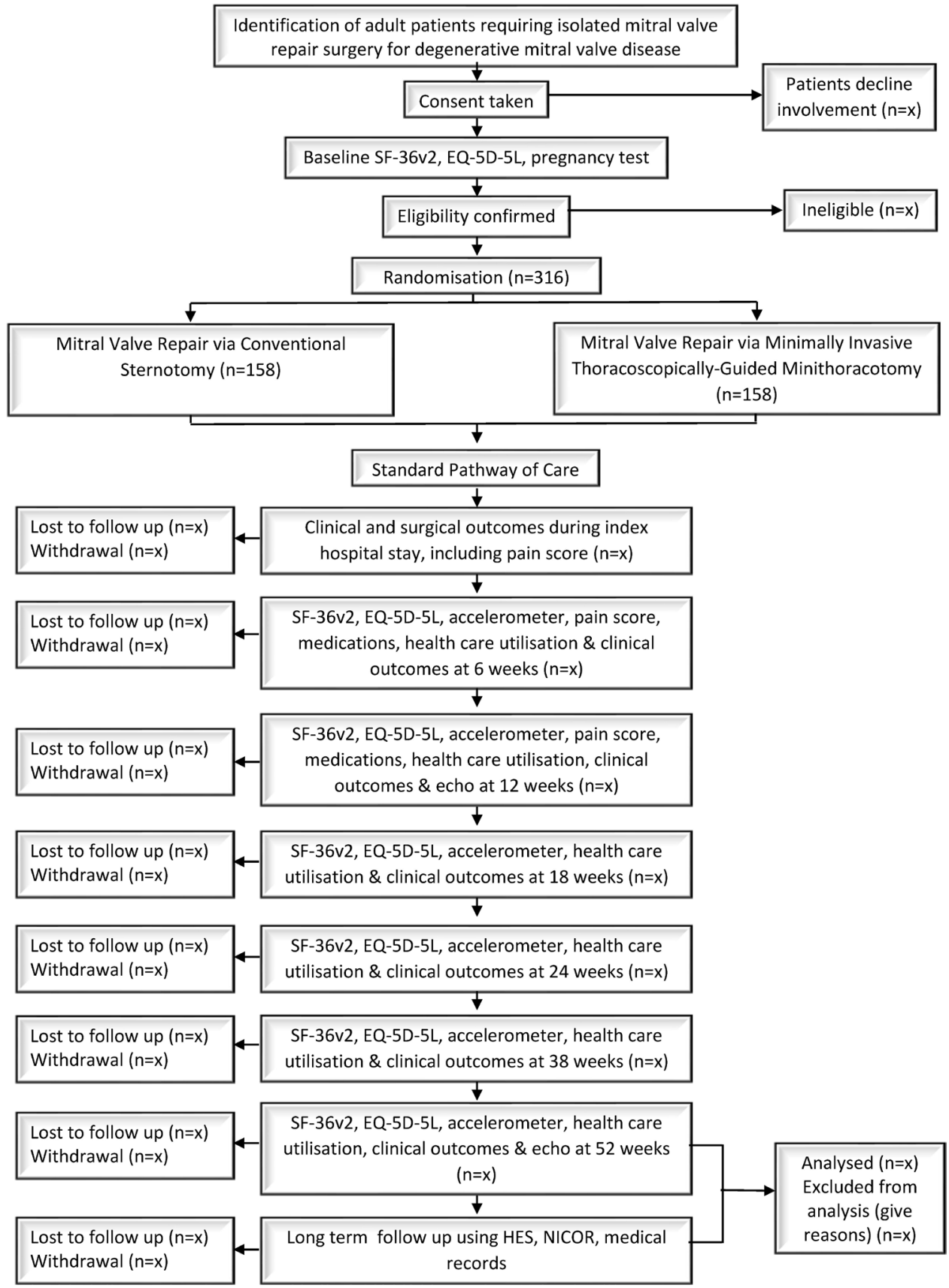

Figure 1 UK Mini Mitral Trial flow diagram. EQ-5D-5L, EuroQol 5 Dimension 5 Level; SF-36v2, Short Form Health Survey.

outcomes (with more than two categories) will be analysed using baseline-category logit model, while log-rank test and frailty modelling will be used to analyse time-toevent outcomes. Analysis of all outcomes with repeated measures on the same patient will account for intrapatient correlation. Multiple imputation for secondary outcomes will be used if necessary to sensitise incomplete data. All outcomes will also be described using simple statistics to facilitate interpretation and communication of findings. Secondary analysis will also include hierarchical modelling of patients, surgeons and centres, to provide an estimate of treatment effect at each of these levels.

Each question within the SF-36v2 physical functioning scale will be scored based on the RAND scoring system. ${ }^{42}$
The average of the per-patient scale scores (assuming each question carries equal weight) will provide the primary outcome for physical functioning at 12 weeks.

\section{Economic analysis}

The economic evaluation will include both a within trial economic evaluation to estimate the incremental cost per QALY gained at 52 weeks and a model based economic evaluation to estimate cumulative costs and QALYs and incremental cost per QALY gained over a patient lifetime. The analysis will take the perspective of the UK NHS.

The within trial analysis will consist of a microcosting of the intervention (minimally invasive minithoracotomy) and usual care (conventional sternotomy). The cost of 
each surgical procedure will be derived from information captured in the case report form and obtained from the team at clinical centres. The subsequent use of NHS services will be captured through patient questionnaires to record use of subsequent primary and secondary care services.

A state transition model will enable extrapolation of costs and outcomes beyond 52 weeks for the predicted lifetime of participants. Data used to populate the model will come from the within trial analysis and external data sources systematically derived from the literature as per best practice guidance. ${ }^{52}$ External data will help identify health state utilities for events occurring long term and not captured within the trial. This will include HES data, validated using medical records and the National Adult Cardiac Surgical Database, adjudicated by an independent expert panel. The independent expert panel will assess which use of health services is attributable to sequelae from index surgery, or underlying disease condition (eg, surgical revision or complication, worsening mitral disease or infection) and/or which is unrelated.

\section{Trial conduct and governance}

The Trial Management Group oversees all day-to-day aspects to ensure that the protocol is adhered to, ensuring patient safety and data integrity; they meet monthly. The IDMEC report to the TSC and provide advice on the ongoing conduct and safety; they meet 6 monthly. The TSC, where independent members are in the majority, provides overall supervision; they meet 6 monthly.

\section{Patient and public involvement}

Meetings with cardiac surgeons and cardiologists in the UK and the USA established the need for, and feasibility of, a definitive trial. Ideas were discussed with patient members of the South Cleveland Heart Fund in 2014 and again in 2015. All patients fully supported the trial and informed the primary outcome; their view that returning to usual activities was the best way to compare the two techniques matched that of surgeons and cardiologists. A patient is a member of the TSC and the team have committed to sharing the results of this trial with patients.

\section{Blinding}

SF-36v2 and EQ-5D-5L assessments are completed over the telephone by a central blinded assessor.

All echocardiograms are sent to an independent Core Laboratory for assessment by a senior echocardiographer, who reports these without knowledge of the intervention.

\section{ETHICS AND DISSEMINATION}

The trial team carefully thought through potential issues and aimed to address these in the trial design. This study does not include patients who will lack capacity, nor minors. This trial is not based in an emergency setting. The trial is conducted in accordance with the protocol, the principles of Good Clinical Practice, and the favourable ethical opinion. All patients provide written informed consent prior to participation, and for their data to be used in future research.

The main issue is a relatively new cardiac procedure, though all cardiac surgery has inherent risks. To mitigate this, surgery will only be performed by highly experienced consultant cardiac surgeons who will have performed at least 50 operations of the same type prior to the start of their involvement. The trial will require the collection and storage of sensitive personal data. All data are handled in accordance with the appropriate legislation. We will only publish deidentified aggregated data.

The results of the trial are expected to fill an important and large gap in the international literature. We expect that the trial results will inform an important revision of national and international. We will publish the findings in peer-reviewed journals, and disseminate results to patients, and the international clinical community.

\section{Author affiliations \\ ${ }^{1}$ Newcastle Clinical Trials Unit, Newcastle University, Newcastle upon Tyne, UK ${ }^{2}$ Department of Anthropology, Durham University, Durham, UK \\ ${ }^{3}$ The Lancashire Cardiac Centre, Blackpool Teaching Hospitals NHS Foundation Trust, Blackpool, UK \\ ${ }^{4}$ Health Economics Group, Population Health Sciences Institute, Newcastle University, Newcastle, UK \\ ${ }^{5}$ Cardiothoracic Surgery, South Tees Hospitals NHS Foundation Trust, Middlesbrough, UK \\ ${ }^{6}$ Cardiothoracic Surgery, King's College Hospital NHS Foundation Trust, London, UK ${ }^{7}$ Department of Cardiovascular Sciences and NIHR Leicester Biomedical Research Unit in Cardiovascular Medicine, University of Leicester, Leicester, UK \\ ${ }^{8}$ Cardiothoracic Surgery, Royal Infirmary of Edinburgh, Edinburgh, UK \\ ${ }^{9}$ Cardiothoracic Surgery, University Hospitals Plymouth NHS Trust, Plymouth, UK \\ ${ }^{10}$ Cardiothoracic Surgery, Imperial College Healthcare NHS Trust, London, UK \\ ${ }^{11}$ Cardiothoracic Surgery, University Hospitals Bristol NHS Foundation Trust, Bristol, UK \\ ${ }^{12}$ Bristol Heart Institute, University of Bristol, Bristol, UK \\ ${ }^{13}$ Cardiac Surgery, Liverpool Heart and Chest Hospital NHS Foundation Trust, Liverpool, UK \\ ${ }^{14}$ Cardiothoracic Surgery, Liverpool Heart and Chest Hospital NHS Foundation Trust, Liverpool, UK}

\section{Twitter Vipin Zamvar @VipinZamvar}

Acknowledgements We are grateful to the IDMEC, TSC, and the independent expert panel for their support of the trial. We would like to acknowledge the considerable work being undertaken by Principal Investigators and members of the site teams, and at Newcastle Clinical Trials Unit in support of this trial.

Contributors EA and JZ conceived the idea for the trial. EA, RHM, HCH, ASK, GJM, JZ and LV codesigned the trial, secured funding from NIHR HTA and wrote the protocol. EA is chief Investigator. $\mathrm{RHM}$ and $\mathrm{HCH}$ provide methodological input and oversee NCTU activity. ASK leads statistical aspects and analysis. LV leads health economic aspects and analysis, supported by CF-G. LM manages the trial. RG leads the core laboratory. GJM provides ongoing clinical and academic contributions. JZ, AW, GL, RD, AG, SK, VZ, RP, CL, MD-H, RC, HAV, FC, SS, MC, MB, GN, PPP, OW, PM, BHK, MDP, ADM and DP are surgeons in the trial and who have contributed to ongoing trial design and the Statistical Analysis Plan. This paper was drafted from the approved version of the protocol, version 5.0, 18th June 2020; all authors commented and amended drafts of the paper and approved the final manuscript.

Funding This work is supported by a National Institute for Health Research (NIHR) Health Technology Assessment (HTA) Programme (Ref: 14/192/110). The UK Mini Mitral Trial is sponsored by South Tees Hospitals NHS Foundation Trust. 
Competing interests LV was a member of the NIHR Health Technology Assessment Clinical Evaluation and Trials Panel from 2015 to 2018.

Patient and public involvement Patients and/or the public were involved in the design, or conduct, or reporting, or dissemination plans of this research. Refer to the Methods section for further details.

\section{Patient consent for publication Not required.}

Provenance and peer review Not commissioned; peer reviewed for ethical and funding approval prior to submission.

Open access This is an open access article distributed in accordance with the Creative Commons Attribution 4.0 Unported (CC BY 4.0) license, which permits others to copy, redistribute, remix, transform and build upon this work for any purpose, provided the original work is properly cited, a link to the licence is given, and indication of whether changes were made. See: https://creativecommons.org/ licenses/by/4.0/.

\section{ORCID iDs}

Rebecca H Maier http://orcid.org/0000-0002-7350-3288

Adetayo S Kasim http://orcid.org/0000-0002-0411-3059

Luke Vale http://orcid.org/0000-0001-8574-8429

Gavin J Murphy http://orcid.org/0000-0003-0976-1482

Vipin Zamvar http://orcid.org/0000-0003-2447-828X

Massimo Caputo http://orcid.org/0000-0001-7508-0891

Cristina Fernandez-Garcia http://orcid.org/0000-0002-7113-225X

Helen C Hancock http://orcid.org/0000-0002-1494-8551

Enoch Akowuah http://orcid.org/0000-0002-2429-3579

\section{REFERENCES}

1 McNeely CA, Vassileva CM. Long-Term outcomes of mitral valve repair versus replacement for degenerative disease: a systematic review. Curr Cardiol Rev 2015;11:157-62.

2 Gaur P, Kaneko T, McGurk S, et al. Mitral valve repair versus replacement in the elderly: short-term and long-term outcomes. $J$ Thorac Cardiovasc Surg 2014;148:1400-6.

3 Coutinho GF, Correia PM, Antunes MJ. Concomitant aortic and mitral surgery: to replace or repair the mitral valve? J Thorac Cardiovasc Surg 2014;148:1386-92.

4 Candan O, Ozdemir N, Aung SM, et al. Effect of mitral valve repair versus replacement on left ventricular rotational deformation: a study with speckle tracking echocardiography. $J$ Heart Valve Dis 2013;22:651-9.

5 Markar SR, Sadat U, Edmonds L, et al. Mitral valve repair versus replacement in the elderly population. J Heart Valve Dis 2011;20:265-71.

6 Chikwe J, Goldstone AB, Passage J, et al. A propensity scoreadjusted retrospective comparison of early and mid-term results of mitral valve repair versus replacement in octogenarians. Eur Heart $J$ 2011;32:618-26.

7 lung B, Baron G, Butchart EG, et al. A prospective survey of patients with valvular heart disease in Europe: the Euro heart survey on valvular heart disease. Eur Heart J 2003;24:1231-43.

8 Keogh B, Kinsman R. Fifth national adult cardiac surgical database report improving outcomes for patients, 2003The Society of Cardiothoracic Surgeons of Great Britain and Ireland. Available: https://scts.org/_userfiles/resources/5thBlueBook2003.pdf

9 SCTS Blue Book. Cardiac surgery audit data bluebook, the Society for cardiothoracic surgery (SCTS) blue book.

10 Nishimura RA, Otto CM, Bonow RO, et al. 2017 AHA/ACC focused update of the 2014 AHA/ACC guideline for the management of patients with valvular heart disease: a report of the American College of Cardiology/American heart association Task force on clinical practice guidelines. Circulation 2017;135:e1159-95.

11 Kirmani BH, Jones SG, Malaisrie SC, et al. Limited versus full sternotomy for aortic valve replacement. Cochrane Database Syst Rev 2017:4:CD011793.

12 Culliford AT, Cunningham JN, Zeff RH, et al. Sternal and costochondral infections following open-heart surgery. A review of 2,594 cases. J Thorac Cardiovasc Surg 1976;72:714-26.

13 Zacharias A, Habib RH. Factors predisposing to median sternotomy complications. deep vs superficial infection. Chest 1996;110:1173-80.

14 Rabindranauth P, Burns JG, Vessey $\Pi$, et al. Minimally invasive coronary artery bypass grafting is associated with improved clinical outcomes. Innovations 2014;9:421-6.
15 Shrestha M, Krueger H, Umminger J, et al. Minimally invasive valve sparing aortic root replacement (David procedure) is safe. Ann Cardiothorac Surg 2015;4:148-53.

16 Rojas SV, Avsar M, Khalpey Z, et al. Minimally invasive off-pump left ventricular assist device exchange: anterolateral thoracotomy. Artif Organs 2014;38:539-42.

17 Ko K, de Kroon TL, Post MC, et al. Minimally invasive mitral valve surgery: a systematic safety analysis. Open Heart 2020;7:e001393.

18 McClure RS, Athanasopoulos LV, McGurk S, et al. One thousand minimally invasive mitral valve operations: early outcomes, late outcomes, and echocardiographic follow-up. J Thorac Cardiovasc Surg 2013;145:1199-206.

19 Cohn LH, Adams DH, Couper GS, et al. Minimally invasive cardiac valve surgery improves patient satisfaction while reducing costs of cardiac valve replacement and repair. Ann Surg 1997;226:421-6.

20 Deutsch M-A, Krane M, Schneider L, et al. Health-Related quality of life and functional outcome in cardiac surgical patients aged 80 years and older: a prospective single center study. J Card Surg 2014;29:14-21

21 Moscarelli M, Fattouch K, Gaudino M, et al. Minimal access versus sternotomy for complex mitral valve repair: a meta-analysis. Ann Thorac Surg 2020;109:737-44.

22 Grant SW, Hickey GL, Modi P, et al. Propensity-matched analysis of minimally invasive approach versus sternotomy for mitral valve surgery. Heart 2019;105:783-9.

23 Grossi EA, Goldman S, Wolfe JA, et al. Minithoracotomy for mitral valve repair improves inpatient and postdischarge economic savings. $J$ Thorac Cardiovasc Surg 2014:148:2818-22.

24 Sündermann SH, Sromicki J, Rodriguez Cetina Biefer $\mathrm{H}$, et al. Mitral valve surgery: right lateral minithoracotomy or sternotomy? A systematic review and meta-analysis. J Thorac Cardiovasc Surg 2014;148:1989-95.

25 Ding C, Jiang D-ming, Tao K-yu, et al. Anterolateral minithoracotomy versus median sternotomy for mitral valve disease: a meta-analysis. $J$ Zhejiang Univ Sci B 2014;15:522-32.

26 Cao C, Gupta S, Chandrakumar D, et al. A meta-analysis of minimally invasive versus conventional mitral valve repair for patients with degenerative mitral disease. Ann Cardiothorac Surg 2013;2:693-703.

27 Modi P, Hassan A, Chitwood WR. Minimally invasive mitral valve surgery: a systematic review and meta-analysis. Eur J Cardiothorac Surg 2008. :34:943-52. Nov.

28 Cheng $\mathrm{DCH}$, Martin J, Lal A, et al. Minimally invasive versus conventional open mitral valve surgery: a meta-analysis and systematic review. Innovations 2011;6:84-103.

29 Speziale G, Nasso G, Esposito G, et al. Results of mitral valve repair for Barlow disease (bileaflet prolapse) via right minithoracotomy versus conventional median sternotomy: a randomized trial. J Thorac Cardiovasc Surg 2011;142:77-83. Jul.

30 Nasso G, Bonifazi R, Romano V, et al. Three-Year results of repaired Barlow mitral valves via right minithoracotomy versus median sternotomy in a randomized trial. Cardiology 2014;128:97-105.

31 Goldstone AB, Atluri P, Szeto WY, et al. Minimally invasive approach provides at least equivalent results for surgical correction of mitral regurgitation: a propensity-matched comparison. $J$ Thorac Cardiovasc Surg 2013;145:748-56.

32 Lamelas J, Sarria A, Santana O, et al. Outcomes of minimally invasive valve surgery versus median sternotomy in patients age 75 years or greater. Ann Thorac Surg 2011;91:79-84.

33 Mazine A, Vistarini N, Ghoneim A, et al. Very high repair rate using minimally invasive surgery for the treatment of degenerative mitral insufficiency. Can J Cardiol 2015;31:744-51.

34 Holzhey DM, Shi W, Borger MA, et al. Minimally invasive versus sternotomy approach for mitral valve surgery in patients greater than 70 years old: a propensity-matched comparison. Ann Thorac Surg 2011:91:401-5

35 Petracek MR, Leacche M, Solenkova N, et al. Minimally invasive mitral valve surgery expands the surgical options for high-risks patients. Ann Surg 2011;254:606-11.

36 Iribarne A, Easterwood R, Russo MJ, et al. A minimally invasive approach is more cost-effective than a traditional sternotomy approach for mitral valve surgery. J Thorac Cardiovasc Surg 2011;142:1507-14.

37 Seeburger J, Borger MA, Falk V, et al. Minimal invasive mitral valve repair for mitral regurgitation: results of 1339 consecutive patients. Eur J Cardiothorac Surg 2008;34:760-5.

38 Yoo JS, Kim JB, Jung S-H, et al. Echocardiographic assessment of mitral durability in the late period following mitral valve repair: minithoracotomy versus conventional sternotomy. J Thorac Cardiovasc Surg 2014;147:1547-52.

39 Falk V, Cheng DCH, Martin J, et al. Minimally invasive versus open mitral valve surgery: a consensus statement of the International 
Society of minimally invasive coronary surgery (ISMICS) 2010. Innovations 2011;6:66-76.

40 Stone GW, Lindenfeld J, Abraham WT, et al. Transcatheter mitralvalve repair in patients with heart failure. New England Journal of Medicine 2018;379:2307-18.

41 Feldman T, Foster E, Qureshi M, et al. TCT-788 the Everest II randomized controlled trial (RCT): three year outcomes. J Am Coll Cardiol 2012;60:B229-30.

42 Ware JE, Kosinski M, Bjorner JB, et al. User's Manual for the SF-36v2TM Health Survey. 2nd Ed. Lincoln Rl: QualityMetric Incorporated, 2007.

43 van Hout B, Janssen MF, Feng Y-S, et al. Interim scoring for the EQ5D-5L: mapping the EQ-5D-5L to EQ-5D-3L value sets. Value Health 2012;15:708-15.

44 Devlin NJ, Shah KK, Feng Y, et al. Valuing health-related quality of life: an EQ-5D-5L value set for England. Health Econ 2018;27:7-22.

45 Herdman M, Gudex C, Lloyd A, et al. Development and preliminary testing of the new five-level version of EQ-5D (EQ-5D-5L). Qual Life Res 2011;20:1727-36.
46 Esliger DW, Rowlands ANNV, Hurst TL, et al. Validation of the GENEA Accelerometer. Med Sci Sport Exerc 2011;43:1085-93.

47 Pavey T, Gomersall S, Clark B, et al. The validity of the GENEActiv wrist-worn accelerometer for measuring sedentary behaviour in free living. J Sci and Med in Sport 2014;18:e34.

48 Sirichana W, Moore-Gillon CE, Patel MH. Daily physical activity in COPD: quantification by Tri Axial Accelerometry. Am J Respir Crit Care Med 2013;187:A1361 https://www.atsjournals.org/doi/abs/10. 1164/ajrccm-conference.2013.187.1_MeetingAbstracts.A1361

49 Troiano RP, McClain JJ, Brychta RJ, et al. Evolution of accelerometer methods for physical activity research. Br J Sports Med 2014;48:1019-23.

50 Hildebrand M, VAN Hees VT, Hansen BH, et al. Age group comparability of raw accelerometer output from wrist- and hip-worn monitors. Med Sci Sports Exerc 2014;46:1816-24.

51 EQ-5D. Available: https://euroqol.org/

52 NICE. Guide to the methods of technology appraisal; 2013. 